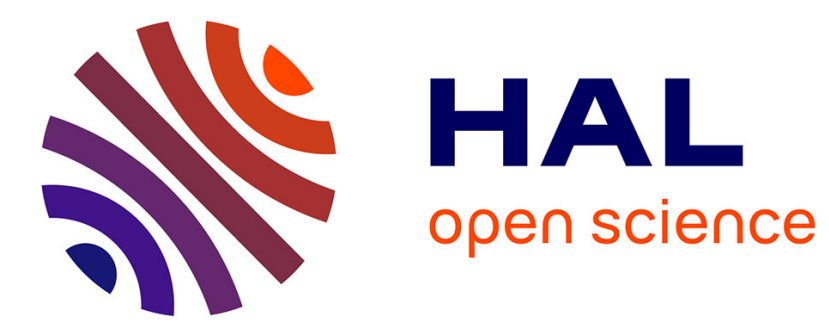

\title{
Broadband magnetic losses in Fe-Si and Fe-Co laminations
}

C Beatrice, C Appino, Olivier de La Barrière, F Fiorillo, C Ragusa

\section{To cite this version:}

C Beatrice, C Appino, Olivier de La Barrière, F Fiorillo, C Ragusa. Broadband magnetic losses in Fe-Si and Fe-Co laminations. IEEE Transactions on Magnetics, 2014, pp.80 - 83 . hal-01100320

\section{HAL Id: hal-01100320 \\ https://hal.science/hal-01100320}

Submitted on 6 Jan 2015

HAL is a multi-disciplinary open access archive for the deposit and dissemination of scientific research documents, whether they are published or not. The documents may come from teaching and research institutions in France or abroad, or from public or private research centers.
L'archive ouverte pluridisciplinaire HAL, est destinée au dépôt et à la diffusion de documents scientifiques de niveau recherche, publiés ou non, émanant des établissements d'enseignement et de recherche français ou étrangers, des laboratoires publics ou privés. 


\title{
Broadband magnetic losses in Fe-Si and Fe-Co laminations
}

\author{
C. Beatrice ${ }^{1}$, C. Appino ${ }^{1}$, O. de la Barrière ${ }^{2 a}$, F. Fiorillo ${ }^{1}$, and C. Ragusa ${ }^{3}$ \\ ${ }^{1}$ Electromagnetics Department, Istituto Nazionale di Ricerca Metrologica (INRIM), Torino, Italy \\ ${ }^{2}$ SATIE, CNRS, UniverSud, 61 av du President Wilson, F-94230 Cachan, France \\ ${ }^{3}$ Dipart. Energia, Politecnico di Torino, C.so Duca degli Abruzzi 24, 10129 Torino, Italy
}

\begin{abstract}
We discuss comprehensive broadband ( $\mathrm{DC}-10 \mathrm{kHz}$ ) investigations on magnetic losses in $\mathrm{Fe}-(3 \mathrm{wt} \%) \mathrm{Si}$ and $\mathrm{Fe}-\mathrm{Co}$ laminations. In this range of frequencies, the prediction of loss is not easy, because skin effect can be quite important. The theoretical approach generally relies on a dynamic hysteresis model in association with a diffusion equation, but it imposes heavy computational burden. We present here a computationally efficient dynamic hysteresis model based on the Dynamic Preisach Model (DPM), by which one can achieve fast and precise solution of the diffusion equation taking into account the hysteretic constitutive equation of the material. This is achieved at a greatly reduced computational cost with respect to the standard DPM. The loss results provided by this simplified model are at all frequencies in very good agreement with the prediction by the full DPM and with the experiments.
\end{abstract}

Index Terms-Magnetic losses, Fe-Si and Fe-Co laminations, Dynamic Preisach Model, Skin effect.

\section{INTRODUCTION}

$\mathrm{T}$ HE PREDICTION of power losses in magnetic laminations up to high frequencies is an important issue in the design of high-speed high-torque density machines [1]. It is, however, a challenging task, because skin effect cannot be neglected and an accurate and computationally efficient model is required for a satisfactory treatment of the losses at high frequencies. Some authors have tried empirical approaches, derived from the Steinmetz equation corrected by appropriate frequency dependent coefficients, to take into account the skin effect [2]. The dependence of these coefficients from frequency is obtained through a linear skin effect formulation, assuming constant material permeability. This approximation proves effective only at low induction levels, far from magnetic saturation. At technically relevant inductions the non-linear hysteretic material behavior must be considered, as put in evidence in [3].

To address the whole problem, significant work has been performed in the literature, aimed at a physically based model, taking into account both skin effect and the non-linear hysteretic constitutive law of the material. The solution of the diffusion equation using a finite element approach, and the modeling of the material constitutive law given by the Static Preisach Model (SPM) has been considered in [4]. The hysteretic $B(H)$ relationship is dealt with using the fixed point method and an iterative algorithm is applied in [5]. Although computationally efficient, thanks to the Everett formalism [6], the SPM has the drawback of not taking into account the role of the domain walls in the magnetization dynamics (i.e. the excess loss contribution). This has been then addressed by means of a dynamic hysteretic relationship, like the viscoustype model [7], or Bertotti's Dynamic Preisach Model (DPM) [8]. Because of its solid physical basis and the fact that it only requires the experimental identification of a single material constant (i.e. the dynamic constant $k_{\mathrm{d}}$ [9]), the DPM has become very popular [10]. The implementation of the DPM into a numerical scheme for solving the diffusion equation of the magnetic field can well take into account skin effect, as discussed in [11] [12], where the fixed point method is used to deal with the non-linearity. Although accurate, this procedure is computationally cumbersome, because the state of every hysteron must be evaluated at each time step [8] on every finite element, until convergence of the iterative scheme is attained.

The purpose of this work is to derive a simplified dynamic hysteresis model based on the DPM and its physical framework. It requires greatly reduced computation time, (the computational burden being approximately that of the SPM). This model and the full DPM are compared with the experimental magnetic energy losses in $0.2 \mathrm{~mm}$ thick Fe-Si and $\mathrm{Fe}-\mathrm{Co}$ laminations, measured in the frequency range $\mathrm{DC}$ $10 \mathrm{kHz}$. It is shown that the prediction by the simplified DPM closely agrees with the experiments and the full DPM simulations, at a drastically reduced computational cost.

\section{Modeling}

\section{A. Computational scheme}

The concept of static magnetic field $H_{\text {stat }}$ is first introduced. It is the field that would give, under quasi-static excitation, the same irreversible polarization $J_{\text {irr }}(t)$ obtained under dynamic conditions through the applied field $H(t)$. Once the static field $H_{\text {stat }}$ is known, the irreversible polarization $J_{\text {irr }}$ can be efficiently computed by application of the SPM. The main question is how to derive the static field $H_{\text {stat }}$ from knowledge of the dynamic magnetic field $H(t)$. In the following, it is shown that a simplified relationship between $H$ and $H_{\text {stat }}$ can be derived in terms of a non-linear first order differential equation in $H_{\text {stat }}$, whose solution can provide the static field $H_{\text {stat }}$ for any given field $H$. This model is summed up in Fig. 1, where the dynamic operator $\boldsymbol{F}$ takes into account the dynamic properties of the hysteretic model. Once $J_{\text {irr }}$ is known, it is added to the reversible component $J_{\text {rev }}$ to obtain the total polarization $J(t)$. The irreversible and reversible density functions are identified using the same technique as in [10].

\section{B. Differential relation between $H$ and $H_{\text {stat }}$}

The dynamic operator $\boldsymbol{F}$ is derived from the DPM under appropriate simplifying assumptions. In the following, the dynamic constant $k_{\mathrm{d}}$ of the DPM is assumed to be known (it is identified according to [9]).

\footnotetext{
${ }^{a}$ Corresponding author, e-mail : barriere@ satie.ens-cachan.fr
} 


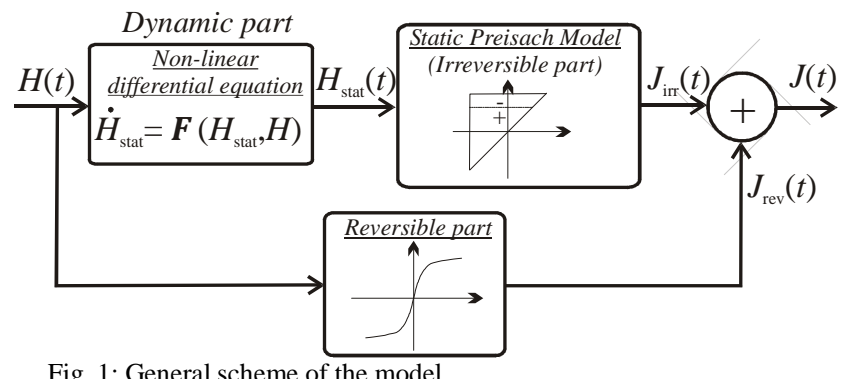

Fig. 1: General scheme of the model

We consider the case of an increasing magnetic field. It has been shown in [13] that the set of switching hysterons in the dynamic Preisach plane $\alpha-\beta$ ( $\alpha$ and $\beta$ being the up and down switching fields, respectively) can be represented as a strip of width $\Delta H$ (Fig. 2), in the interval $[H-\Delta H, H]$. This relates to the fact that under dynamic conditions hysteron switching takes time. On the contrary, in the Static Preisach Model, the state of each hysteron is assumed to instantaneously switch from -1 to 1 . All hysterons in the $S^{+}$area are positively saturated, whereas the ones in the $S^{-}$area are negatively saturated. A function $\phi$, defined in [8], represents the state of each hysteron in the interval $[-1,1]$. An example is reported in Fig. 2 where the $\phi$ function depends only on $\alpha$, because we are on the ascending branch of the field $H$. Since the hysterons in the interval $[H-\Delta H, H]$ are commuting and display an intermediate state, $\Phi$ makes a smooth transition between -1 and +1 .

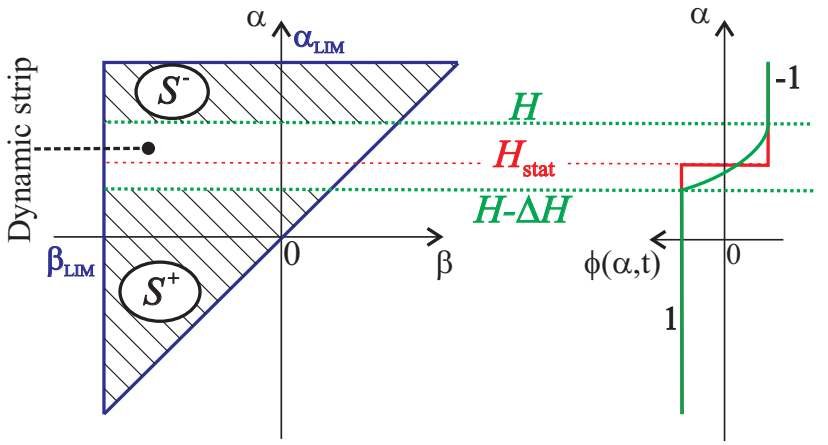

Fig. 2: Representation of the dynamic and static Preisach planes providing the same irreversible polarization contribution $J_{\text {irr }}$

In the SPM the boundary between the positively and negatively saturated hysterons is instead a horizontal line of coordinate $H_{\text {stat }}$, and the function $\phi$ is therefore a step like one (Fig. 2). Assuming $H$ is increasing, the following function $\mu(\alpha)$ is introduced [13]:

$$
\mu(\alpha)=J_{\mathrm{s}} \int_{\beta_{\mathrm{LIM}}}^{\alpha} p_{\text {irr }}(\alpha, \beta) d \beta,
$$

where $p_{\text {irr }}(\alpha, \beta)$ is the normalized irreversible part of the Preisach density function, and $J_{\mathrm{s}}$ is the saturation polarization. According to the definition of $H_{\text {stat }}$, the following relation holds

$$
\int_{H-\Delta H}^{H} \mu(\alpha) \phi(\alpha, t) \mathrm{d} \alpha=\int_{H-\Delta H}^{H_{\text {stat }}} \mu(\alpha) \mathrm{d} \alpha-\int_{H_{\text {stat }}}^{H} \mu(\alpha) \mathrm{d} \alpha .
$$

Equation (2) defines the static field at each instant of time, given the state function $\phi$ and the width $\Delta H$. According to the DPM theory [8], we express the flux derivative as $\dot{\phi}=k_{\mathrm{d}} \cdot(H-\alpha)$. From the time derivative of (2) we consequently obtain

$$
\dot{H}_{\text {stat }}=\frac{k_{d}}{2 \mu\left(H_{\text {stat }}\right)} \int_{H-\Delta H}^{H}(H-\alpha) \mu(\alpha) \mathrm{d} \alpha,
$$

representing the dynamical part of the model. The solution of (3) requires some simplifying assumptions, as explained in the following.

Assumption of constant $\mu$ function on the strip. On the interval $[H-\Delta H, H]$, the function $\mu$ is assumed to be constant, equal to its mean value (the validity of this hypothesis is discussed later). Equation (2) therefore becomes

$$
\int_{H-\Delta H}^{H}[1+\phi(\alpha, t)] d \alpha=2 \cdot\left(H_{\text {stat }}-H+\Delta H\right)
$$

and (3) provides

$$
\dot{H}_{\text {stat }}=\frac{k_{\mathrm{d}}}{4} \Delta H^{2} .
$$

Assumption of local constant time derivative of the magnetic field. In this case, as shown in [13], the $\phi$ function is given by the parabolic function

$$
\phi(\alpha, t)=-1+\frac{2}{\Delta H^{2}}(H-\alpha)^{2},
$$

where the strip width $\Delta H$ has been introduced by taking into account that $\phi(H-\Delta H, t)=1$. Introducing (6) in (4), the width $\Delta H$ is obtained:

$$
\Delta H=\frac{3}{2}\left(H-H_{\text {stat }}\right) .
$$

Eq. (7) is further introduced in (5) providing the following differential equation

$$
\dot{H}_{\text {stat }}=\operatorname{sign}\left[H(t)-H_{\text {stat }}(t)\right] \frac{9}{16} k_{\mathrm{d}}\left[H(t)-H_{\text {stat }}(t)\right]^{2},
$$

where sign $= \pm 1$ according to the ascending or descending direction of the magnetic field. Equation (8) eventually defines the dynamic operator $\boldsymbol{F}$ of Fig. 1. This equation can be numerically solved by a Crank-Nicholson method, applying periodic conditions on the static field $H_{\text {stat }}$. The involved computational burden of the model is around that required by SPM.

We have introduced this simplified DPM in a numerical scheme for the computation of the magnetic field and flux distribution in the lamination. The results are compared with those provided by the full DPM and the DC $10 \mathrm{kHz}$ experiments.

\section{EXPERIMENTAL}

The measurements were performed on commercial nonoriented $\mathrm{Fe}-(3 \mathrm{wt} \%) \mathrm{Si}$ and $\mathrm{Fe}_{50} \mathrm{Co}_{50}$ sheets. There thickness $d$ is equal to $0.194 \mathrm{~mm}$ and $0.201 \mathrm{~mm}$, respectively. The measurements were performed up to $10 \mathrm{kHz}$ using the 200 turn Epstein frame, with the test strips cut half along and half transverse to the rolling direction, according to the measuring standard IEC 60404-10 [14]. The DC normal magnetization curve was obtained up to magnetic saturation by means of the 
point-by-point method (calibrated fluxmeter Lake-Shore mod. 480). The hysteresis loops and losses were measured versus frequency for several defined peak polarization values $J_{\mathrm{p}}$ by means of a hysteresisgraph-wattmeter with digital control of the flux waveform. The form factor of the secondary voltage was kept at all frequencies within the interval $\mathrm{FF}=1.1107 \pm$ $0.4 \%$. The primary winding was supplied, depending on $J_{\mathrm{p}}$, and frequency either by a $200 \mathrm{~W}$ NF HSA4101 or a $1 \mathrm{~kW} \mathrm{NF}$ 4510 power amplifier, driven by an Agilent 33210A function generator.

For each material, the constant of the DPM $k_{\mathrm{d}}$ has been obtained measuring hysteresis loops at $50 \mathrm{~Hz}, 100 \mathrm{~Hz}$ and $200 \mathrm{~Hz}$, for peak inductions of $0.5 \mathrm{~T}, 1 \mathrm{~T}$, and $1.5 \mathrm{~T}$, and by fitting the related excess loss values. It is provided, together with the main physical parameters of the investigated laminations, in Table I.

TABLE I

DATA ON THE TWO MATERIALS USED FOR MEASUREMENTS

\begin{tabular}{ccc}
\hline \hline & Fe-Si & Fe-Co \\
\hline Composition & $\mathrm{Fe}-(3 \mathrm{wt} \%) \mathrm{Si}$ & $\mathrm{Fe}_{49} \mathrm{Co}_{49} \mathrm{~V}_{2}$ \\
Annealing & $780^{\circ} \mathrm{C}, 2$ hours & $820^{\circ} \mathrm{C}, 10$ hours \\
Thickness $d(\mathrm{~mm})$ & 0.194 & 0.201 \\
Density $\delta\left(\mathrm{kg} / \mathrm{m}^{3}\right)$ & 7650 & 8120 \\
Conductivity $\sigma(\mathrm{S} / \mathrm{m})$ & $1.92 \cdot 10^{6}$ & $2.27 \cdot 10^{6}$ \\
Constant $k_{\mathrm{d}}$ of the DPM & 700 & 1100 \\
$\left(\mathrm{~m}^{-1} \mathrm{~s}^{-1}\right)$ & & \\
\hline \hline
\end{tabular}
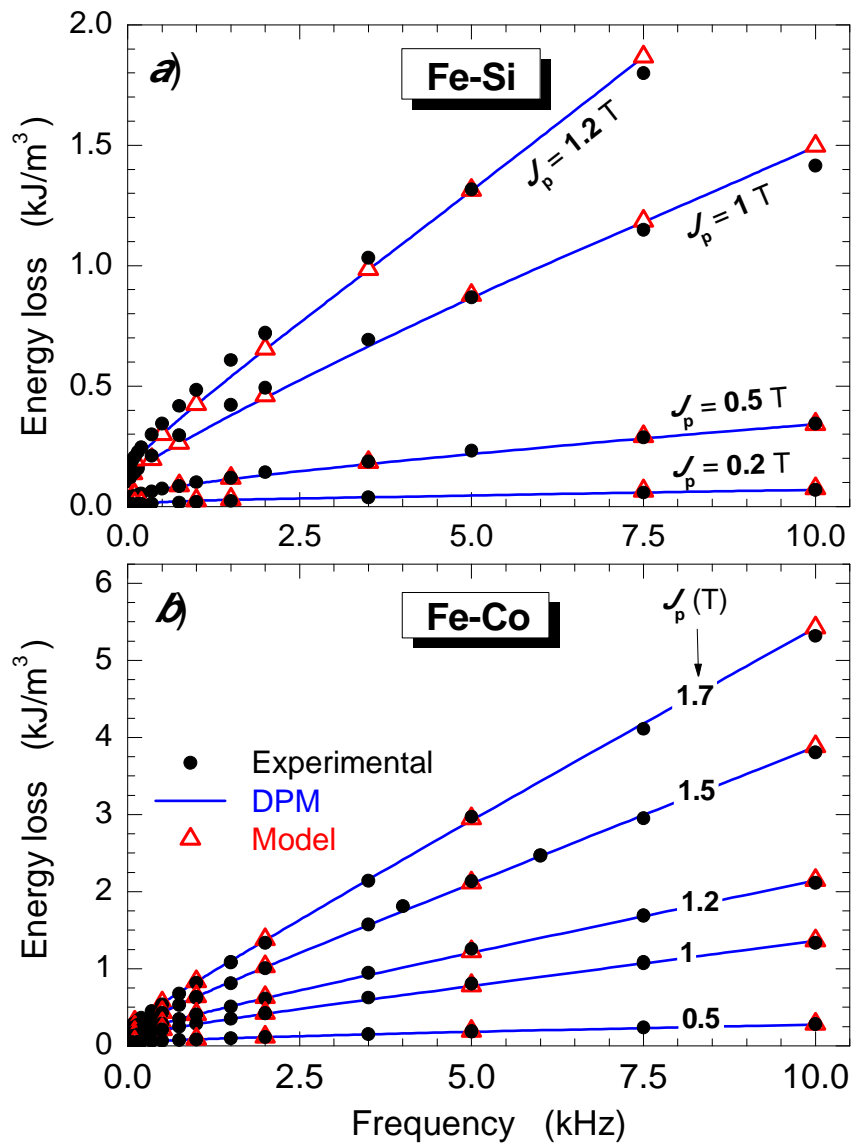

Fig. 3 - Energy loss versus frequency at different polarization values $J_{\mathrm{p}}$ (full symbols: experiments, solid line: full DPM, open triangle: simplified DPM, a) case of $\mathrm{Fe}-\mathrm{Si}$ laminations, and b) case of $\mathrm{Fe}-\mathrm{Co}$ laminations.

\section{A. Loss results}

Application of simplified and full DPMs to the energy loss measurements in the $\mathrm{Fe}-\mathrm{Si}$ laminations are shown in Fig. $3 \mathrm{a}$. The results obtained in the Fe-Co sheets are presented in Fig. 3b. Fe-Co, with its high saturation polarization $\left(J_{\mathrm{s}}=2.35 \mathrm{~T}\right)$, permits one to perform broadband measurements up to pretty high inductions. Remarkably, the measured losses are excellently described by the abovementioned models, including the high-frequency results, which are considerably influenced by the skin effect.

\section{B. Measured and computed cycles}

Hysteresis cycles, experimentally obtained and computed by the DPM and the simplified model, are compared in Fig. 4.

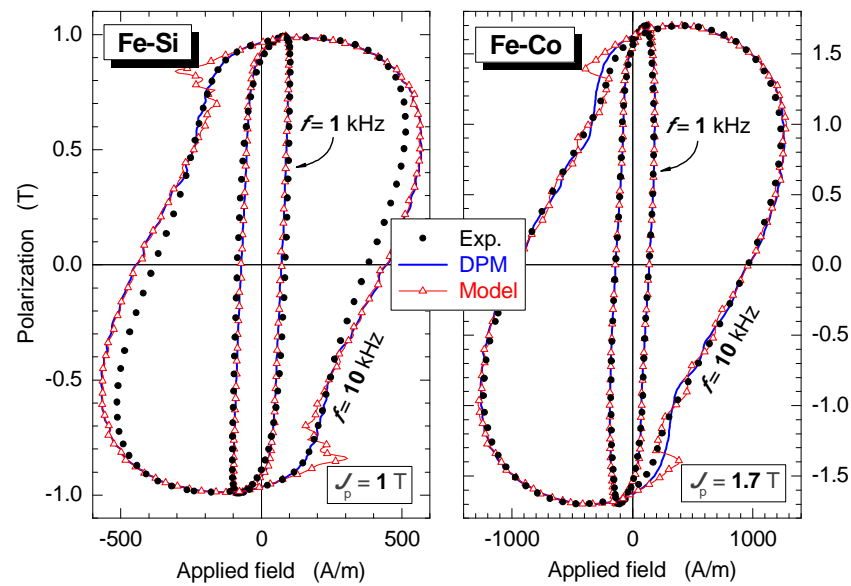

Fig. 4 - Dynamic cycles in Fe-Si $\left(J_{\mathrm{p}}=1.0 \mathrm{~T}\right)$ and Fe-Co $\left(J_{\mathrm{p}}=1.7 \mathrm{~T}\right)$ sheets at $1 \mathrm{kHz}$ and $10 \mathrm{kHz}$. The experimental loops (symbols) are compared with the prediction by the full (solid line) and simplified (line plus symbols) DPMs.

The dynamic loops are accurately reproduced at the frequency $f=1 \mathrm{kHz}$ in both materials. Minor discrepancies are found at $f=10 \mathrm{kHz}$, where the induction profile in the lamination becomes highly inhomogeneous. This is understood as due to the fact that at the highest frequencies, the dynamic strip of the DPM (Fig. 2) can be quite large, and the assumption of constant $\mu$ function becomes untenable. But the loop area is still predicted with acceptable accuracy.

\section{B. Influence of the skin effect}

In the absence of appreciable skin effect, we assume that the mean polarization $J(t)$ and the local one are the same, and the classical loss is simply obtained, for sinusoidal induction of peak value $B_{\mathrm{p}}$

$$
W_{\text {class }}\left(J_{\mathrm{p}}, f\right)=\frac{\pi^{2}}{6} \cdot \sigma d^{2} B_{\mathrm{p}}^{2} f .
$$

In this case, the hysteresis and excess loss contributions can be found by direct inversion of the hysteresis model presented in this paper. Energy loss calculations in Fe-Co using the model with and without skin effect are compared in Fig. 5 with the experimental values. While the need for considering the skin effect in loss modeling is apparent, we see that when this is ignored, different kinds of discrepancies arise at low and high inductions. Thus, for $J_{\mathrm{p}}=0.5 \mathrm{~T}$ the conventional loss model, where uniform induction is assumed in the lamination crosssection, overestimates the actual loss, while the opposite occurs at high inductions (e.g. $J_{\mathrm{p}}=1.7 \mathrm{~T}$ ). 


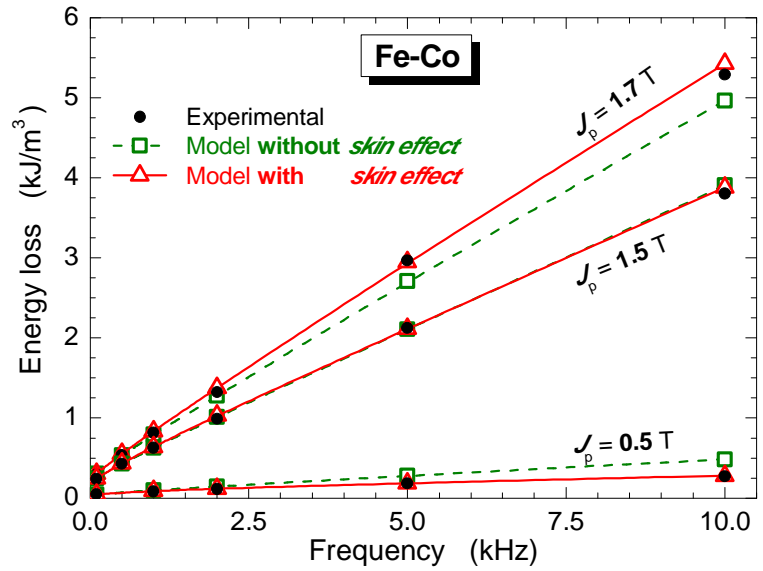

Fig. 5 - The prediction of energy losses by the standard model ignoring skin effect overestimates the actual figures at low inductions and underestimates them at high inductions.

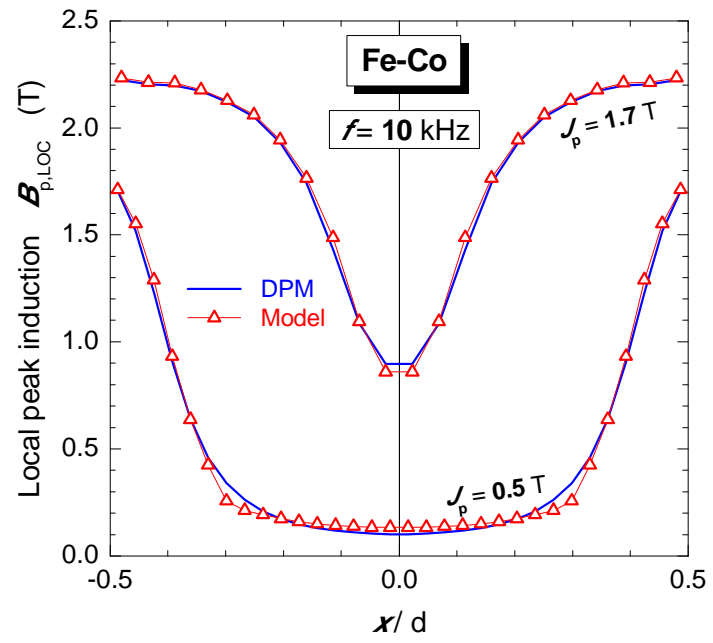

Fig. 6 - Calculated profile of the peak induction $B_{\mathrm{p}, \mathrm{LOC}}(x)$ across the lamination thickness $(-d / 2 \leq x \leq d / 2, d=0.201 \mathrm{~mm})$ in Fe-Co $(f=10 \mathrm{kHz})$. Low and high average peak polarization values $\left(J_{\mathrm{p}}=0.5 \mathrm{~T}\right.$ and $\left.J_{\mathrm{p}}=1.7 \mathrm{~T}\right)$ are considered.

It turns then out that no differences arise at intermediate inductions, as shown for $J_{\mathrm{p}}=1.5 \mathrm{~T}$ in Fig. 6. This evolution follows from the progressively increasing depth of the magnetically saturated layer on increasing the $J_{\mathrm{p}}$ value. This imposes a corresponding evolution of the induction profile across the lamination thickness, as illustrated in Fig. 6 for $J_{\mathrm{p}}=$ $0.5 \mathrm{~T}$ and $J_{\mathrm{p}}=1.7 \mathrm{~T}(f=10 \mathrm{kHz})$. We see here that for $J_{\mathrm{p}}=$ $1.7 \mathrm{~T}$ the saturated region penetrates a fraction of the sample cross-sectional area while for $J_{\mathrm{p}}=0.5 \mathrm{~T}$, the profile has the freedom to adjust close to the predictable behavior of the local peak induction $B_{\mathrm{p}, \mathrm{LOC}}(x)(-d / 2 \leq x \leq d / 2)$ in a constant permeability material.

\section{CONCLUSIONS}

We have discussed DC-10 kHz loss measurements carried out for several peak polarization values in $0.2 \mathrm{~mm}$ thick $\mathrm{Fe}-\mathrm{Si}$ and Fe-Co laminations. They are assessed by means of a computationally efficient dynamic hysteresis model based on the Dynamic Preisach Modeling (DPM), here proposed and implemented. This model has been introduced in a fixed point iterative procedure taking into account magnetic field diffusion across the lamination depth. The calculated energy losses and dynamic hysteresis loops and their evolution with frequency are in excellent agreement with the experiments and with the full DPM prediction, while showing strongly reduced computation time.

\section{ACKNOWLEDGMENT}

This work was partly supported by the MIUR-PRIN2009 Project MEA 2009LKCT3E "Advanced characterization and modeling of magnetic materials for the More Electric Aircraft" and the e-méca project of the French ANR ("Agence Nationale de la Recherche').

\section{REFERENCES}

[1] H-W Cho, K-J Ko, J-Y Choi, H-J Shin, and S-M Jang, "Rotor Natural Frequency in High-Speed Permanent-Magnet Synchronous Motor for Turbo-Compressor Application," IEEE Trans. Magn., vol. 47, no. 10, pp. 4258-4261, 2011.

[2] A. Boglietti, A. Cavagnino, M. Lazzari, and M. Pastorelli, "Predicting iron losses in soft magnetic materials with arbitrary voltage supply: an engineering approach," IEEE Trans. Magn., vol. 39, no. 2, pp. 981989, 2003.

[3] P. Rasilo, A. Belahcen, and A. Arkkio, "Importance of Iron-Loss Modeling in Simulation of Wound-Field Synchronous Machines," IEEE Trans. Magn., vol. 48, no. 9, pp. 2495-2504, 2012

[4] C. Appino, G. Bertotti, O. Bottauscio, F. Fiorillo, P. Tiberto, D. Binesti, J.P. Ducreux, M. Chiampi, and M. Repetto, "Power losses in thick steel laminations with hysteresis," J. Appl. Phys., vol. 79, no. 8, pp. 45754577, 1996.

[5] F.I. Hantila, G. Preda, and M. Vasiliu, "Polarization method for static fields," IEEE Trans. Magn., vol. 36, no. 4, pp. 672-675, 2000.

[6] I. Mayergoyz, "Mathematical models of hysteresis," IEEE Trans. Magn., vol. 22, no. 5, pp. 603-608, 1986.

[7] S.E. Zirka, Y.I. Moroz, P. Marketos, and A.J. Moses, "A ViscousType Dynamic Hysteresis Model as a Tool for Loss Separation in Conducting Ferromagnetic Laminations," IEEE Trans. Magn., vol. 41, no. 3, pp. 1109$1111,2005$.

[8] G. Bertotti, "Dynamic Generalization of the Scalar Preisach Model of Hysteresis," IEEE Trans. Magn., vol. 28, no. 5, pp. 2599-2601, 1992.

[9] L.L. Rouve, T. Waeckerle, A. Kedous-Lebouc, and J.L. Coulomb, "Determination of the parameter $\mathrm{k}$ of the generalized dynamic Preisach model," IEEE Trans. Magn., vol. 32, no. 3, pp. 1124-1127, 1996.

[10] L.R. Dupre, O. Bottauscio, M. Chiampi, M. Repetto, and J.

Melkebeek, "Modeling of electromagnetic phenomena in soft magnetic materials under unidirectional time periodic flux excitations," IEEE Trans. Magn., vol. 35, no. 5, pp. 4171-4184, 1999.

[11] O. Bottauscio, M. Chiampi, C. Ragusa, and M. Repetto, "A Fixed Point Iteration H Scheme in the Solution of Hysteretic Electromagnetic Field Problems," in Studies in Applied Electromagnetics and Mechanics. Non-linear electromagnetic systems, V. Kose and J. Sievert, Ed.: IOS Press, 1998, pp. 449-454.

[12] V. Basso, G. Bertotti, O. Bottauscio, M. Chiampi, F. Fiorillo, M. Pasquale, and M. Repetto, ""Power losses in magnetic laminations with hysteresis: finite element modelling and experimental validation," J. Appl. Phys. vol. 81 pp. 5606 - 5608, 1997.

[13] G. Bertotti, "Generalized Preisach model for the description of hysteresis and eddy current effects in metallic ferromagnetic materials," $J$. Appl. Phys., vol. 69, no. 8, pp. 4608-4610, 1991

[14] IEC Standard Publication 60404-10, "Methods of measurement of magnetic properties of magnetic steel sheet and strip at medium frequencies (Geneva, IEC Central Office, 1988). 